\title{
The effect of magnetization pinning on the spectrum of spin waves in magnonic antidot waveguides
}

\author{
J. W. Kłos ${ }^{1}{ }^{*}$ and D. Kumar ${ }^{2}$, J. Romero-Vivas ${ }^{1}$, \\ H. Fangohr ${ }^{3}$, M. Franchin ${ }^{3}$, M. Krawczyk ${ }^{1}$, A. Barman ${ }^{2}$ \\ ${ }^{1}$ Faculty of Physics, Adam Mickiewicz University in Poznań, \\ Umultowska 85, Poznań, 61-614, Poland; \\ ${ }^{2}$ Department of Condensed Matter Physics and Material Sciences, \\ S. N. Bose National Centre for Basic Sciences, \\ Block JD, Sector III, Salt Lake, Kolkata 700 098, India; \\ ${ }^{3}$ Engineering and the Environment, \\ University of Southampton, Southampton SO17 1BJ, UK.
}

(Dated: September 27, 2012)

\begin{abstract}
We study the spin-wave spectra in magnonic antidot waveguides (MAWs) for two limiting cases (strong and negligible) of the surface anisotropy at the ferromagnet/air interface. The MAWs under investigation have the form of a thin stripe of permalloy with a single row of periodically arranged antidots in the middle. The introduction of a magnetization pinning at the edges of the permalloy stripe and the edges of antidots is found to modify the spin-wave spectrum. This effect is shown to be necessary for magnonic gaps to open in the considered systems. Our study demonstrates that the surface anisotropy can be crucial in the practical applications of MAWs and related structures and in the interpretation of experimental results in one- and two-dimensional magnonic crystals. We used three different numerical methods i.e. plane waves method (PWM), finite difference method and finite element method to validate the results. We showed that PWM in the present formulation assumes pinned magnetization while in micromagnetic simulations special care must be taken to introduce pinning.
\end{abstract}

PACS numbers: $75.75 .+\mathrm{a}, 76.50 .+\mathrm{g}, 75.30 . \mathrm{Ds}, 75.50 . \mathrm{Bb}$ 


\section{INTRODUCTION}

A relatively new and rapidly emerging field of physics, ${ }^{1-4}$ magnonics is mainly aimed at exploiting the properties of spin waves (SWs) to harness them for technological applications in a variety of fields. These include spintronics, microwave systems, metamaterials for electromagnetic waves, and other magnonic devices using spin waves for carrying and processing information. Except for microwave technology, the other three fields are new and their development is at the initial stage. ${ }^{2,5-8}$

The possibility of tailoring metallic magnetic materials with nanoscale precision provides a tool for miniaturization and shaping the dispersion of high-frequency spin waves. This can be done by periodic modulation in magnonic crystals (MCs), ${ }^{1,9}$ the magnetic analogues of photonic crystals, in which frequency gaps open in the spin-wave spectrum for certain structural and material parameters. Not until recently have the first one-dimensional ${ }^{10}$ (1D) and two-dimensional $^{11}$ (2D) bi-component MCs been realized at the nanoscale. In particular, 2D antiots lattices can be easily prepared experimentally by creating periodic arrays of holes in ferromagnetic films. These systems have been intensively studied in the last few years, on various length scales and considering various antidot geometry and crystallographic arrangement. ${ }^{12-16}$

This paper is focused on the boundary conditions imposed on the dynamic components of the magnetization vector and their effect on the spectrum of spin waves in magnonic waveguides. These boundary conditions ${ }^{17}$ are additional to the electromagnetic ones, which describe the degree of freedom of the magnetization vector at the edges of the ferromagnetic material. The effect of the boundary conditions on the spectrum of SWs in uniform thin films has been investigated broadly. ${ }^{17-21}$ The general form of boundary conditions at external faces of the ferromagnetic plane proposed by Guslienko ${ }^{18}$ takes into account both dipolar pinning and pinning induced by uniaxial surface anisotropy:

$$
\boldsymbol{M} \times\left(\lambda_{\mathrm{ex}}^{2} \frac{\partial \boldsymbol{M}}{\partial n}+\frac{2 K_{\mathrm{s}}}{\mu_{0} M_{\mathrm{s}}^{2}}\left(\boldsymbol{M} \cdot \boldsymbol{n}_{\mathrm{a}}\right) \boldsymbol{n}_{\mathrm{a}}+\boldsymbol{H}_{\mathrm{dm}} 2 c\right)=0,
$$

where $\lambda_{\text {ex }}$ and $M_{\mathrm{s}}$ denote exchange length and magnetization saturation, respectively. The symbol $\frac{\partial}{\partial n}$ stands for directional derivative along the normal to the face. The uniaxial surface anizotropy is defined by its strength $K_{\mathrm{s}}$ and orientation $\boldsymbol{n}_{\mathrm{a}}$. The demagnetizing field $\boldsymbol{H}_{\mathrm{dm}}$ taken at the given face determines the contribution of dipolar pinning. $\boldsymbol{H}_{\mathrm{dm}}$ depends on the thickness $2 c$ and in-plane sizes of the system $R$ ( e.g. stripe width). In was shown ${ }^{18}$ that for 
small systems $\left(\sqrt{R u}<\lambda_{\text {ex }}\right)$ the magnetization pinning can be achieved only in the presence of strong surface anisotropy. Therefore in exchange limit the Rado-Weertman boundary condition $^{17}$, which neglect the dipolar pinning, are sufficient.

The research comparing the role of different types of boundary conditions has not been conducted so far in periodic waveguides, in a form of antidot lattices. In this structures the interfaces with air can play an important role in the formation of magnonic bands. ${ }^{22,23}$ Only free boundary conditions are assumed in the vast majority of papers dealing with periodic waveguides. Thus, there is a gap in the research, which we attempt to fill in with this study. We decide to explore two limiting cases: free boundary conditions and strong pinning using different computational techniques. Note that the surface anisotropy field (second term in the brackets in Eq. (1)) depend monotonously on the $K_{\mathrm{s}}$. As a result, the logarithmic derivate of the components of dynamical magnetization $\left(\frac{\partial}{\partial n} m_{\alpha}\right) / m_{\alpha}$ ( $\alpha$ indicates the Cartesian components) taken on the side faces of waveguide has also monotonous dependence on $K_{\mathrm{s}}$ in the regime of linear dynamics ${ }^{18}$ and approaches the values $\pm \infty$ (pinned boundary conditions) and $\ll 1$ (unpinned boundary conditions) for high and low values of $K_{\mathrm{s}}$, respectively. Therefore these two extreme cases limit the area of investigation for the impact of boundary conditions on the spin waves spectra in confined geometry of waveguide close to exchange regime.

In this paper we study the magnonic band structure in waveguides, a basic element of any magnonic device. ${ }^{24,25}$ Waveguides for exchange spin waves have been recently investigated theoretically with the use of micromagnetic simulations; ${ }^{26-28}$ periodic waveguides have been demonstrated to have filter properties due to the folding effect and the opening of magnonic gaps in the spin-wave spectrum. ${ }^{29,30}$ Here we investigate a periodically modulated waveguide with a series of antidots in the center. Aware of the fact that the periodicity of the waveguide can be realized in many different ways - by width or shape corrugation, or by applying a specific magnetic field ${ }^{28-30}$ — we are confident that the fundamental features of this quasi-1D periodic system are conserved and the conclusions drawn for the model considered will be of general nature.

We show that a magnetization pinning introduced at the edges of the waveguide can significantly change its spin-wave spectrum. To crosscheck our results we perform calculations based on different methods: micromagnetic simulations and the plane wave method (PWM). These techniques have already been successfully used for the interpretation of experimental 
data obtained for systems of various geometry in the formulation used here.

We show that the pining is intrinsic for PWM at the interface of magnetic/nonmagnetic material. For micromagnetic calculations the magnetic moments are not forced to be pinned by default. We introduce pining by placing on the interface thin layer for which the dynamics of magnetization was frozen with the amplitude of precession equals to zero.

The paper is organized as follows. In Section II we describe the structure under investigation and the calculation methods used. In Section III we explain the effects that the boundary conditions imposed on the dynamic magnetization components at the edges of the ferromagnetic material have on the magnonic spectrum. Our results are summarized in the closing Section IV.

\section{THE WAVEGUIDE STRUCTURE AND THE CALCULATION METHODS}

The magnonic waveguide under consideration is shown in Fig. 1. It has the form of a thin and infinitely long permalloy stripe with a single row of square holes disposed periodically along the central line. A bias magnetic field is applied along the stripe and assumed to be strong enough $\left(H_{0}=1.01 \mathrm{~T}\right)$ to saturate the sample. The following parameter values are assumed in all the calculations: saturation magnetization in Py $0.8 \times 10^{6} \mathrm{~A} / \mathrm{m}$, exchange constant $1.3 \times 10^{-11} \mathrm{~J} / \mathrm{m}$, and gyromagnetic ratio $175.9 \mathrm{GHz} / \mathrm{T}$.

We use three methods of calculating the dispersion of spin waves in the permalloy MAW: the finite difference method, the finite element method and the PWM, with OOMMF, ${ }^{31}$ $\mathrm{Nmag}^{32}$ and a home-developed Fortran code, respectively. All the methods solve the LandauLifshitz-Gilbert equation:

$$
\begin{aligned}
\frac{\partial \boldsymbol{M}(\boldsymbol{r}, t)}{\partial t}= & \gamma \mu_{0} \boldsymbol{M}(\boldsymbol{r}, t) \times \boldsymbol{H}_{\mathrm{eff}}(\boldsymbol{r}, t) \\
& -\frac{\alpha}{M_{\mathrm{s}}}\left(\boldsymbol{M}(\boldsymbol{r}, t) \times \frac{\partial \boldsymbol{M}(\boldsymbol{r}, t)}{\partial t}\right),
\end{aligned}
$$

with a first term on the right hand side related to the torque inducing precession and the second one describing the damping process. The symbols: $\gamma$ and $\alpha$ denotes gyromagnetic ratio and damping constant, respectively. The effective field $\boldsymbol{H}_{\text {eff }}$ consists of the following terms:

$$
\boldsymbol{H}_{\mathrm{eff}}(\boldsymbol{r}, t)=\boldsymbol{H}_{0}+\boldsymbol{H}_{\mathrm{dm}}(\boldsymbol{r}, t)+\boldsymbol{H}_{\mathrm{ex}}(\boldsymbol{r}, t)
$$


with $\boldsymbol{H}_{0}, \boldsymbol{H}_{\mathrm{dm}}(\boldsymbol{r}, t)=\boldsymbol{H}_{\mathrm{dm}}(\boldsymbol{r})+\boldsymbol{h}_{\mathrm{dm}}(\boldsymbol{r}, t), \boldsymbol{H}_{\mathrm{ex}}(\boldsymbol{r}, t)$ being external field, demagnetizing field and exchange field, respectively.

\section{A. Micromagnetic simulation}

The micromagnetic simulations (OOMMF, Nmag) are performed in two steps. The magnetic ground state is obtained first. We let the magnetization evolve in the presence of damping to reach the static equilibrium orientation. In the next step, with damping neglected $(\alpha=0)$, a small pulse of magnetic field was applied in the shape as given by Eq. (4) with a small amplitude (which guarantees the linear regime of spin dynamics). After recording the magnetization in each mesh point for each time step, Fourier transformation is

performed in the time and space domains to obtain the SW dispersion, i.e. the wave-vector dependence of the SW frequency. ${ }^{33,34}$

The excitation signal used to study the dynamics is of the form:

$$
\begin{aligned}
h_{z}(t, x, y)= & h_{\mathrm{z}}^{0} \operatorname{sinc}\left(2 \pi f_{\text {cut }}\left(t-t_{0}\right)\right) \\
& \times \operatorname{sinc}\left(k_{\text {cut }}\left(x-\frac{x_{\max }}{2}\right)\right) \\
& \times \sum_{n=1}^{N} \sin \left(n \pi \frac{y}{y_{\max }}\right),
\end{aligned}
$$

where sinc function is taken in the form: $\operatorname{sinc}(\theta)=\sin (\theta) / \theta$. The strength of the signal is defined by $\mu_{0} h_{\mathrm{z}}^{0}=5 \mathrm{mT}$. The parameter $f_{\text {cut }}=490 \mathrm{GHz}$ sets the upper limit of frequencies of spin waves exited by the sinc-like pulse. An offset $t_{0}=50 \mathrm{ps}$ was given to avoid the high spikes close to the $f_{\text {cut }}$ in the frequency domain of the signal. The $k_{\text {cut }}$ is a wave number cutoff defined later. The symbols: $x_{\max }=3 \mu \mathrm{m}$ and $y_{\max }=15 \mathrm{~nm}$ denote the sizes of sample. The summation in (4) was done for $N=30$ subdivisions.

The benefits for using such a signal and the procedure to obtain the desired dispersion relation are described in further detail in Ref. 34. In the case of an antidot lattice the effect of convolution of the periodic array of holes will be observed in the wave-vector domain. A wave-vector cutoff $k_{\text {cut }}$, which is an odd multiple of half the Brillouin zone (BZ) length (here $3 \pi / a)$ may be used to mitigate this effect. Also, in order to generate both symmetric and anti-symmetric wave-fronts a suitable dependence (which, here, is a sum of symmetric and anti-symmetric excitations along the width) on $y$ has been applied to the signal. 
The OOMMF simulations are performed with 1D periodic boundary conditions applied along the $x$-axis with the formulation defined in Ref. 35 . A $1.5 \mathrm{~nm}$ mesh is used in these simulations. The correctness of the assumed discretization was verified by comparing the results with those of simulations using a $0.5 \mathrm{~nm}$ mesh.

The micromagnetic simulations do not assume by default any torque acting on the external interfaces (numerical calculations are limited to the magnetic medium only). Therefore the spins are precessing freely on the systems boundaries with dipolar effects taken fully into account.

We can force the pinning in all mesh cells located on the interfaces between magnetic and non-magnetic materials (see the pinning area red-coloured in Fig. 1). It can be done by freezing the magnetization dynamics: $\frac{\partial}{\partial t} \boldsymbol{M}(\boldsymbol{r}, t)=0$ with initial conditions: $M_{\mathrm{z}}(\boldsymbol{r}, t=0)=0$ and $M_{\mathrm{y}}(\boldsymbol{r}, t=0)=0$ at the beginning of the second stage of calculation when the system managed to reach the ground state. From Landau-Lifshitz-Gilbert Eq. (3) follows that the initial condition for $\mathrm{z}$ - and $\mathrm{y}$-components of magnetization will be sustain while the condition $M_{\mathrm{z}}(\boldsymbol{r}, t=0)=0$ is by keep in pinning layer. We checked that for strong external filed we used $\left(\mu_{0} H_{0}=1.01 \mathrm{~T}\right)$ the magnetization in the ground state is uniform and co-linear with the direction of $H_{0}$ even in the vicinity of the interfaces.

Because of the use of the finite difference method in OOMMF simulations, space is discretised into small cuboids. Nmag uses the finite element method, in which, in contrast, the modeled object is discretised on a tetrahedral mesh. In general this allows for better modelling of arbitrarily shaped objects, but for the considered antidot waveguide, this does not provides an advantage because our simulating object consists bascally of orthogonal walls. For the mesh creation, we use the open source generator 'NETGEN'. When creating the unstructured mesh, care must be taken on providing the software with a proper value of the maximum-mesh-size parameter. Only when this value is small enough, it is possible to calculate the exchange and magnetostatic fields with reasonable accuracy. Unfortunately, as the maximum-mesh-size parameter decreases, the number of tetrahedral elements increase, making the computing time and memory demands increase as well. The one way to partially overcome this problem is to use adaptive mesh with the smaller cell sizes in the vicinity of antidots edges. We must select a value below the exchange length, which in the case of permalloy is $5.1 \mathrm{~nm}$. Therefore, we selected a maximum size of $4.5 \mathrm{~nm}$ for the edge length of all tetrahedra to achieve accurate results. However, the average edge length was about 
$2.5 \mathrm{~nm}$ with standard deviation equals $0.6 \mathrm{~nm}$. It was decided to not use periodic boundary conditions in Nmag simulations but instead use a finite segment of this waveguide of length 1.8 micrometer, containing 120 repetitions of the unit cell. The waveguide is surrounded by non-magnetic material which does not have to be discretised. This is because Nmag used a hybrid finite elements/boundary elements method to calculate the magnetostatic contribution.

The steps to obtain the dispersion relation are the same as in OOMMF. As a first step, a high value of the Gilbert damping parameter is chosen and the system is evolved under the external field to find the energy-minimizing configuration of the system. This state is used as the starting point during the second part. Now damping is neglected and the system is excited with a pulse containig a broad frequency range. Using the Fourier transform, the resonating values of $(k, \omega)$ are obtained as local maxima. These values constitute the dispersion diagram.

\section{B. Plane waves method}

In the PWM, periodic Bloch conditions are applied both along the MAW axis and in the direction perpendicular to this axis. An artificial periodicity in the $y$ direction creates a periodic series of non-interacting copies of the original waveguide-this is the supercell approach. ${ }^{36}$ We used the supercell (marked in Fig. 1 by dashed line). The assumed periods are $15 \mathrm{~nm}$ (antidot period) and $100 \mathrm{~nm}$ (artificial supercell) along the $x$ - and $y$-axes, respectively.

The antidots and spacer areas were filled with artificial material characterized by high values of volume anisotropy field and extremely low value of magnetization saturation what squeezed the magnetization dynamics in this regions (effect of the low magnetisation saturation) and shifted the frequencies of spurious modes appearing in results into very high frequency range (impact of the high volume anisotropy field). Note that according to Landau-Lifshitz equation the increase of the effective field in the artificial material (as a result of big volume anisotropy) will also decrease the amplitudes of dynamical magnetization if one wants to keep the spin wave frequency constant. We have made sure that the assumed $65 \mathrm{~nm}$ waveguide spacing is sufficient to neglect the interactions between adjacent copies. We plotted the dispersion relation in $\Gamma$ - $Y$ direction (i.e. for propagation direc- 
tion perpendicular to the waveguides axis). The branches that we obtained were flat what confirmed the localization of spin waves in Y-direction and the lack of crosstalks between adjacent copies of waveguides. We also check the amplitude of spin waves in the spacers separating waveguides, which occurred to be canceled. The canceling of spin dynamics in the air gaps (spacers and antidot areas) results in magnetization pinning at the interface with magnetic material. I order to simulate the system of planar geometry with partially pinned magnetization on the interfaces with nonmagnetic material one can artificially change the in-plane sizes of the system from $R$ to effective one $R_{\mathrm{eff}}$ to achieve non-zero value of dynamical magnetization when the position $r$ coincide with $R{ }^{19}$. We used this procedure to perform PWM calculations in dipolar-exchange regime for $2 \mathrm{D}$ antidot lattices of holes. ${ }^{37}$

We used in the calculation Eq. (3) with damping effects neglected. We are considering the magnetization dynamics in the linear approximation only. We are assuming that the magnetization precesses around $\mathrm{x}$-axis in a cone with small angle (as it is presented in the Fig. 1) with angular frequency $\omega$. Under this assumption we can write:

$$
\begin{aligned}
& M_{\mathrm{x}}(\boldsymbol{r}) \approx M_{\mathrm{s}}, \\
& M_{\mathrm{y}}(\boldsymbol{r}, t)=m_{\mathrm{y}}(\boldsymbol{r}) e^{i \omega t}, \\
& M_{\mathrm{z}}(\boldsymbol{r}, t)=m_{\mathrm{z}}(\boldsymbol{r}) e^{i \omega t} .
\end{aligned}
$$

The exchange term can be expressed as: ${ }^{38}$

$$
\boldsymbol{H}_{\mathrm{ex}}=\nabla \lambda_{\mathrm{ex}}^{2} \nabla \boldsymbol{M}(\boldsymbol{r}, t)
$$

and directly deviated form the Heisenberg model. ${ }^{39}$

To describe the demagnetizing field for a periodic slab of finite thickness we used the ideas proposed by Kaczer ${ }^{40}$ and then developed in Ref. 41 where each component of (static $\boldsymbol{H}_{\mathrm{dm}}(\boldsymbol{r})$ and dynamic $\left.\boldsymbol{h}_{\mathrm{dm}}(\boldsymbol{r}, t)\right)$ demagnetizing field is depending, in general, on the spatial distribution of all components of magnetization. The components of the static and dynamic demagnetizing fields within the linear approximation taken into account are:

$$
\begin{aligned}
H_{\mathrm{dm}, \mathrm{x}}(\boldsymbol{r})= & -\sum_{\boldsymbol{G}} M_{\mathrm{s}}(\boldsymbol{G})\left(\frac{G_{\mathrm{x}}}{G}\right)^{2}(1-C(z, G)) \\
& \times e^{-i\left(\boldsymbol{G} \cdot \boldsymbol{r}_{\|}\right)}, \\
h_{\mathrm{dm}, \mathrm{z}}(\boldsymbol{r}, t)= & \sum_{\boldsymbol{G}}\left[-m_{\mathrm{z}}(\boldsymbol{G}) C(z,|\boldsymbol{G}+\boldsymbol{k}|)\right. \\
& \left.+i m_{\mathrm{y}}(\boldsymbol{G}) \frac{\left|k_{\mathrm{y}}+G_{\mathrm{y}}\right|}{|\boldsymbol{G}+\boldsymbol{k}|} S(z,|\boldsymbol{G}+\boldsymbol{k}|)\right]
\end{aligned}
$$




$$
\begin{aligned}
h_{\mathrm{dm}, \mathrm{y}}(\boldsymbol{r}, t)= & \times e^{i \omega t} e^{-i\left((\boldsymbol{G}+\boldsymbol{k}) \cdot \boldsymbol{r}_{\|}\right)}, \\
& \times\left(-m_{\mathrm{y}}(\boldsymbol{G}) \frac{\left(k_{\mathrm{y}}+G_{\mathrm{y}}\right)^{2}}{|\boldsymbol{G}+\boldsymbol{k}|^{2}}\right. \\
& \times(1-C(z,|\boldsymbol{G}+\boldsymbol{k}|)) \\
& \left.+i m_{\mathrm{z}}(\boldsymbol{G}) \frac{\left|k_{\mathrm{y}}+G_{\mathrm{y}}\right|}{|\boldsymbol{G}+\boldsymbol{k}|} S(z,|\boldsymbol{G}+\boldsymbol{k}|)\right] \\
& \times e^{i \omega t} e^{-i\left((\boldsymbol{G}+\boldsymbol{k}) \cdot \boldsymbol{r}_{\|}\right)},
\end{aligned}
$$

where $\boldsymbol{G}=\left[G_{x}, G_{y}\right]$ and $\boldsymbol{r}_{\|}=[x, y]$ are $2 \mathrm{D}$ reciprocal lattice vector and position vector in real space. The symbols: $M_{s}(\boldsymbol{G})$ and $m_{\alpha}(\boldsymbol{G})$ denote the coefficient of Fourier expansion for magnetization saturation $M_{\mathrm{s}}\left(\boldsymbol{r}_{\|}\right)=\sum_{\boldsymbol{G}} M(\boldsymbol{G}) e^{-i\left(\boldsymbol{G} \cdot \boldsymbol{r}_{\|}\right)}$and periodic part of Bloch functions: $m_{\alpha}\left(\boldsymbol{r}_{\|}\right)=\sum_{\boldsymbol{G}} m_{\alpha}(\boldsymbol{G}) M(\boldsymbol{G}) e^{-i\left((\boldsymbol{G}+\boldsymbol{k}) \cdot \boldsymbol{r}_{\|}\right)}, \alpha=y, z$, where $\boldsymbol{k}$ is wave vector. The functions $C(z, \kappa)$ and $S(z, \kappa)$ are defined as:

$$
\begin{aligned}
C(z, \kappa) & =\frac{\sinh (z \kappa)}{\sinh (c \kappa)+\sinh (c \kappa)}, \\
S(z, \kappa) & =\frac{\cosh (z \kappa)}{\sinh (c \kappa)+\sinh (c \kappa)},
\end{aligned}
$$

where $2 c$ is the thickness of MAW (in z-direction). The demagnetizing fields do not change a lot across the slab except the regions in the close vicinity of the external surfaces (note that the structure is uniform in $z$-direction). Therefore we assumed that all fields: $\boldsymbol{H}_{\mathrm{dm}}\left(\boldsymbol{r}_{\|}\right)$ and $\boldsymbol{h}_{\mathrm{dm}}\left(\boldsymbol{r}_{\|}, t\right)$ are independent on $z$-coordinate by taking its values from the top of the slab. This simplification allowed us to consider the system as a $2 \mathrm{D}$ one.

In the linearization procedure we take advantage of the assumption: $m_{\alpha}(\boldsymbol{r}) \ll M_{\mathrm{s}}, \alpha=$ $y, z$ and dropped all small terms non-linear with respect to $m_{\alpha}(\boldsymbol{r})$. Then after applying the Fourier transformation we were able to convert the linearized differential equations for $m_{y}(\boldsymbol{r}), m_{z}(\boldsymbol{r})$ into the set of algebraic equation in the form of eigen-problem with $m_{y}(\boldsymbol{G}), m_{z}(\boldsymbol{G})$ as eigen-vectors and $\omega$ playing the role of eigen-frequency.

We checked that the sufficient convergence for the presented dispersion plots (Fig. 3) is achieved for 11x91 plane waves propagating in x- and y-direction, respectively (described by different x- and y-component of reciprocal vectors $\boldsymbol{G}$ ). The details of the PWM, its supercell formulation and the application of this technique are available in the literature. ${ }^{9,36,37,41}$ 


\section{THE EFFECT OF PINNING ON THE MAGNONIC SPECTRUM}

Figure 2 shows the magnonic band structure obtained form the OOMMF and Nmag simulations. The spin-wave spectrum is very rich, with a clear evidence of periodicity and folding effects. Three repetitions of the BZ, delimited by vertical solid lines, are considered. Free boundary conditions for the dynamic components of the magnetization vector (unpinned magnetization) at the edges of Py were used in these calculations. The lack of pinning is confirmed by the mode profiles (bottom of Fig. $2 \mathrm{a}$ and $2 \mathrm{~b}$ ) computed with the OOMMF and Nmag, where the nonzero values of $\left|m_{z}\right|^{2}$ at the air/Py interfaces are observed. The profiles for lowest modes for the frequencies marked by red horizontal lines were presented. The profiles calculated in OOMMF and Nmag show some differences in the distribution of the amplitude $\left|m_{z}\right|^{2}$ along the waveguide. The maps of $\left|m_{z}\right|^{2}$ manifest also the lack periodicity in x-direction what is unexpected for Bloch function. This behavior is a result of superposition of Bloch waves having the same frequencies with a different k-numbers. Note that in micromagnetic simulations the spatial distribution of $\left|m_{z}\right|^{2}$ contains all contributions form different bands intersecting the same frequency level. This bands (eigenmodes) can be populated differently depending on: (1) the way how the system was excited and (2) the peculiarities of the computational methods (finite differences - OOMMF and finite elements - Nmag). However the amplitudes $\left|m_{z}\right|^{2}$ both in Figs. 2a and 2b preserve the one important property related to the mode quantization by constriction in y-direction - the finite width of the waveguide. One can notice that the successive modes (denoted by I, II, III, IV) having the increased number of horizontal nodal lines. Surprisingly, the results obtained by the PWM is different. The PWM spectra are shown in Fig. 3 (red dashed lines). The bands are seen to be shifted up in the frequency scale, and the modes seem less numerous.

In search of explanation of this discrepancy we calculated the profiles of the dynamic components of the magnetization vector in the PWM. The colored maps in Fig. 3, bottom, represent the modulus $\left|m_{\mathrm{z}}\right|^{2}$ of the $z$ component for a number of lowest-frequency modes; blue and red correspond to low and high values of $\left|m_{\mathrm{z}}\right|^{2}$, respectively. In all the modes in question the magnetization is pinned at the Py/air interfaces (thin white lines). Therefore, we will show that the main reason for the discrepancy between the results of PWM and the micromagnetic simulation are the different boundary conditions applied in these two methods. 
Unfortunately, no extension of the PWM method has been developed yet to allow for unpinned magnetization at the interfaces with nonmagnetic material. We can extend the micromagnetic simulations, though, imposing specific boundary conditions on the magnetization vector $\boldsymbol{M}$. The procedure described in the previous section allows to achieve $\boldsymbol{m}=0$ (i.e. pinning of the magnetization $\boldsymbol{M}$ ) at the interfaces with non-magnetic material. Figure 3, top panel, shows the results of the OOMMF simulations (gray lines) and, superimposed, the PWM data. The agreement between the OOMMF and PWM results is satisfactory now, and the effect of the pinning on the magnonic spectrum of the MAW can be explained in detail.

The changes in the spin-wave spectrum resulting from the introduction of pinning are relatively simple in uniform thin films. The main difference is the occurrence of an extra mode, uniform across the film thickness, in the case of unpinned surfaces; surface-localized modes (surface spin waves) can occur, too. ${ }^{21,42}$ The frequencies of the higher modes for pinned and unpinned surfaces are quite similar. As we have shown already, the changes in a MAW are more significant and complex. Many additional modes are seen to occur in the MAW spectrum calculated for unpinned magnetization (see Fig. 2) compared to those obtained in the pinned case (see Fig. 3). Due to the pinning in the row of antidots some modes existing in unpinned system (the modes with high amplitude of $\left|m_{z}\right|^{2}$ in center of MAW - see modes I and III in Fig. 2) can appear in pinned system. The pinning in the center of the MAW reduces the degrees of freedom of the spin waves and practically divides the waveguide into two parallel sub-waveguides weakly coupled through the barrier formed by the antidot series. This is due to the small edge-to-edge distance between neighboring antidots, which results in a minor crosstalk between the spin waves propagating in the two sub-waveguides. The confinement of the modes increases their separation on the frequency scale in the case of MAW with pinned magnetization. Moreover, due to the minor interaction between the sub-waveguides, the eigenstates are almost degenerate for frequencies up to 200 $\mathrm{GHz}$ in a wide wavenumber range.

In the absence of pinning, spin waves spread freely over the whole width of the waveguide. This is why the unpinned modes are distributed more densely on the frequency scale. The dispersion branches of some unpinned modes are the reminescent of the continuous parabolas in Fig. 2. In the low-frequency range this behavior is seen in every second band, i.e. the $2^{\text {nd }}, 4^{\text {th }}$ and $6^{\text {th }}$ bands from the BZ center, where a nodal line should appear in the center 
of the MAW. The lower amplitude of the spin waves in the center of the MAW results in a negligible impact of the antidot series on the spin dynamics. The effect of the antidot series on the spin dynamics is similarly small in (1) the dispersion branches of unpinned modes with a nodal line in the center of the MAW (see Fig. 2c), and (2) all the dispersion branches of pinned modes. In spite of this, their frequencies are not equal due to the different boundary conditions at the external edges of the MAW. Therefore, no frequency agreement can be expected between the unpinned and pinned modes in wires of the same width.

Another important property of the magnonic band structure of MAWs found in our study is that the magnetization pinning at the edges of the MAW results in the opening of magnonic gaps (yellow bars in Fig. 3). This means that even MAWs with as little as 5\% air can be used as filters with stop and pass bands. As the first magnonic gap occurs at the border of the BZ, its opening is clearly related to the periodicity of the MAW. However, the second gap (between the $4^{\text {th }}$ and $5^{\text {th }}$ bands) is seen to open inside the BZ. This indicates a different origin of this gap.

The second gap results from the anticrossing between two pairs of modes: modes with no nodal line within each sub-waveguide and modes with a nodal line in each half of the MAW. In other words, the anticrossing occurs between two parabolas (connected with the lower and higher harmonics across the MAW width) of the spin-wave dispersion crossing due to folding to the $1^{\text {st }} \mathrm{BZ}$.

It is worth to notice that the closing of the gaps in the system with unpinned magnetization is due to the presence of additional bands $\left(1^{\text {st }}, 3^{\text {rd }}, 5^{\text {th }}, \ldots\right)$ corresponding to modes with a significant magnetization amplitude in the center of the MAW (see Fig. $2 \mathrm{a}, \mathrm{b}-1^{\text {st }}$ and $3^{\text {rd }}$ mode). These modes are, in fact, more affected by antidots and more separated from each other than the modes with nodal line in the center of MAW but their presence makes the spectrum of the unpinned system denser and results in more effective bands overlapping.

The relatively small width of the gaps in the system with pinned magnetization is due to the less effective impact of antidots on modes with low value of dynamical magnetization in the center of MAW.

The above discussion applies to the low-frequency range, in which the mode quantization is related to the confinement of spin waves between one edge of the waveguide and the central row of antidots. In the high-energy range the following effects can interfere with this simple mechanism: (1) spin waves can be localized between antidots in the central region 
of the MAW; (2) the crosstalk can be much more efficient in the case of short spin waves, which can easily 'leak out' from one sub-waveguide to the other.

Let us discuss in detail the profiles of the dynamic magnetization component $\left|m_{\mathrm{z}}\right|^{2}$ presented in the bottom panel of Fig. 3. Three types of modes can be distinguished by profile: (1) modes (a-h) originating from the first mode of each sub-waveguide (no nodal line inside each sub-waveguide); (2) modes (i-p) related to the second mode in the completely isolated sub-waveguides (one nodal line in the MAW); (3) modes ( $\mathrm{r}-\mathrm{u}$ ), high-frequency excitations localized mostly between antidots in the center of the MAW. The modes are plotted for different BZ points, indicated in the top panel of Fig. 3. The modes in the center of the BZ have no nodal line perpendicular to the MAW axis, while the modes at the edge of the BZ only have one such line in each BZ. At intermediate points the non-zero amplitude oscillates more smoothly along the MAW axis.

In the low-frequency range the spin-wave modes show the following characteristics: (1) modes occur in pairs with in-phase and out-of-phase correlation between excitations in the two sub-waveguides; (2) the frequency difference between the modes in each pair increases with growing frequency; (3) the mode splitting can be suppressed (even for relatively high frequencies) in every second pair of modes at the edge of the BZ, where the nodal line between antidots blocks the crosstalk between sub-waveguides (cf. modes o,p to m,n).

In order to verify the modes profiles calculated using PWM we plotted also some profiles with the aid of micromagnetic simulation (OOMMF). They are present in Fig.3 in a hot colors scale and their frequencies are marked by horizontal lines to show from which bands they collect the contributions. The labels: a-b, e-f and k-l-m-n present what kind of mixture of Bloch states (calculated using PWM) exist in the profiles calculated with the aid of OOMMF.

Helpful for practical realizations of MAWs is the insensitivity of the magnonic gaps to the shape of the antidots until its filling fraction and mirror symmetry of MAW is unchanged. On such a small scale, with a feature size of a few nanometers, the shape can be expected to play a minor role. The situation will be different in the magnetostatic regime, i.e. for smaller wave vectors and larger antidot periods where the demagnetizing field is strongly shape dependent and can affect on spin-wave spectrum in low frequency regime ${ }^{43}$. 


\section{CONCLUSIONS}

We have shown that the boundary conditions for the dynamic components of the magnetization vector at ferromagnetic material/air interfaces are of much importance for the SW spectra in nanoscale magnonic antidot waveguides. Our results demonstrate that the magnetization pinning facilitates the opening of magnonic gaps in magnonic antidot waveguides with air filling fraction even as low as 5\%. This indicates an additional functionality of this type of waveguides as filters with tunable stop and pass bands. Also, our results

show that the pinning will be an important factor to be considered in the interpretation of experimental data obtained for antidot lattices or designing new devices in which the antidot arrangement is periodic in nanoscale. The pinning or unpinning at the interfaces is usually related to the surface magnetic anisotropy, determined by the shape of the atomic orbitals modified at the interfaces by the surrounding material and the reconstruction or relaxation processes. Thus, the surface anisotropy can depend on many factors, such as the interface structure on the atomic or nanometer scale, the strain, the crystallographic structure or the chemical composition. ${ }^{44}$ In two-dimensional systems the investigation of these effects can be regarded as an extension of the research in magnetic bilayers and multilayers, which were in focus at the time of the discovery of the GMR effect. We have also shown that peculiar properties of computational methods often used in the calculations are related to specific boundary conditions for dynamical component of magnetization implicitly assumed in each method.

\section{Acknowledgments}

We acknowledge the financial support from the Department of Science and Technology, Goverment of India (grant nos. INT/EC/CMS (24/233552), SR/NM/NS-09/2007), Department of Infromation Technology, Goverment of India (grant no. 1(7)/2010/M\&C) and the European Community's Seventh Framework Programme FP7/2007-2013 (Grant Agreement no. 233552 for DYNAMAG and Grant Agreement no. 228673 for MAGNONICS). Dheeraj Kumar would like to acknowledge financial support from CSIR - Senior Research Fellowship 
(File ID: 09/575/(0090)/2011 EMR-I)

* Electronic address: klos@amu.edu.pl

1 S. Neusser and D. Grundler, Advanced Materials, 21, 2927 (2009).

2 V. V. Kruglyak, S. O. Demokritov, and D. Grundler, J. Phys. D: Appl. Phys., 43264001 (2010).

3 A. A. Serga, A. V. Chumak, and B. Hillebrands, J. Phys. D: Appl. Phys, 43, 264002 (2010).

4 B. Lenk, H. Ulrichs, F. Garbs, and M. Manzenberg, Physics Reports, 507, 107 (2011).

5 T. Schneider, A. Serga, B. Hillebrands, and M. Kostylev, J. Nanoelectr. Optoelectr. 3, 69 (2008).

6 F. Macia, A. D. Kent, and F. C. Hoppensteadt, Nanotechnology, 22, 095301 (2010).

7 A. Khitun, M. Bao, and K. L. Wang, J. Phys. D: Appl. Phys., 43, 264005 (2010).

8 R. V. Mikhaylovskiy, E. Hendry, and V. V. Kruglyak, Phys. Rev. B, 82, 195446 (2010).

9 M. Krawczyk and H. Puszkarski, Phys. Rev. B 77, 054437 (2008).

10 Z.-K. Wang, V. Li Zhang, H. S. Lim, S. C. Ng, M. Hau. Kuok, S. Jain, and A. O. Adeyeye, ACS Nano 4, 643, 2010.

11 G. Duerr, M. Madami, S. Neusser, S. Tacchi, G. Gubbiotti, G. Carlotti, and D. Grundler, Appl. Phys. Lett. 99, 202502 (2011).

12 Michael J. Pechan, Chengtao Yu, R. L. Compton, J. P. Park, and P. A. Crowell, J. Appl. Phys. 97, 10J903 (2005).

13 S. Neusser, B. Botters, and D. Grundler, Phys. Rev. B 78, 054406 (2008).

14 S. Neusser, G. Durr, H. G. Bauer, S. Tacchi, M. Madami, G. Woltersdorf, G. Gubbiotti, C. H. Back, and D. Grundler, Phys. Rev. Lett. 105, 067208 (2010).

15 S. Tacchi, M. Madami, G. Gubbiotti, G. Carlotti, A. O. Adeyeye, S. Neusser, B. Botters, and D. Grundler, IEEE Trans. Mag. 46, 1440 (2010).

16 C.-L. Hu, R. Magaraggia, H.-Y. Yuan, C. S. Chang, M. Kostylev, D. Tripathy, A. O. Adeyeye, and R. L. Stamps, Appl. Phys. Lett. 98, 262508 (2011).

17 G. T. Rado and J. R. Weertman, J. Phys. Chem. Solids 11, 315 (1959).

18 K. Yu. Guslienko and A. N. Slavin, Phys. Rev. B 72, 014463 (2005).

19 K. Yu. Guslienko, S. O. Demokritov, B. Hillebrands and A. N. Slavin, Phys. Rev. B 66, 132402 (2002). 
20 H. Puszkarski, Surface Science Reports 20, 45 (1994).

21 S. Mamica, H. Puszkarski, and J.-C. S. Lévy, Phys. Status Solidi B 218, 561 (2000).

22 C. Yu, M. J. Pechan, W. A. Burgei, and G. J. Mankey, J. Appl. Phys. 95, 6648 (2004).

23 R. D. McMichael and B. B. Maranville Phys. Rev. B 74, 024424 (2006).

24 V. E. Demidov, M. P. Kostylev, K. Rott, J. Münchenberger, G. Reiss, and S. O. Demokritov, Appl. Phys. Lett. 99, 082507 (2011).

25 P. Clausen, K. Vogt, H. Schultheiss, S. Schäfer, B. Obry, G. Wolf, P. Pirro, B. Leven, and B. Hillebrands, Appl. Phys. Lett. 99, 162505 (2011).

26 V.V. Kruglyak, R.J. Hicken, J. Magn. Magn. Mater. 306, 191 (2006).

27 K.-S. Lee, D.-S. Han, and S.-K. Kim, Phys. Rev. Lett. 102, 127202 (2009).

28 F. S. Ma, H. S. Lim, Z. K. Wang, S. N. Piramanayagam, S. C. Ng, and M. H. Kuok, Appl. Phys. Lett. 98, 153107 (2011).

29 S.-K. Kim, J. Phys. D: Appl. Phys. 43, 264004 (2010).

30 L. Bai, M. Kohda, and J. Nitta, Appl. Phys. Lett. 98, 172508 (2011).

31 M. Donahue and D. G. Porter, OOMMF Users guide, Version 1.0, Interagency Report NISTIR 6376 (NIST, Gaithersburg, 1999) at http://math.nist.gov/oommf/.

32 T. Fischbacher, M. Franchin, G. Bordignon, and H. Fangohr, IEEE Trans. Mag. 43, 2896 (2007).

33 M. Dvornik, V.V. Kruglyak, Phys. Rev. B 84, 140405 (2011).

34 D. Kumar, O. Dmytriiev, S. Ponraj and A. Barman, J. Phys. D: Appl. Phys. 45, 015001 (2012).

35 K. M. Lebecki, M. J. Donahue, and M. W. Gutowski, J. Phys. D: Appl. Phys. 41, 175005 (2008).

36 J. W. Klos, M. Krawczyk, and M. Sokolovskyy, J. Appl. Phys. 109, 07D311 (2011).

37 S. Neusser, H. G. Bauer, G. Duerr, R. Huber, S. Mamica, G. Woltersdorf, M. Krawczyk, C. H. Back, and D. Grundler, Phys. Rev. B 84, 184411 (2011).

38 A. G. Gurevich and G. A. Melkov, Magnetization Oscillations and Waves, Oxford University Press 2008.

39 C. H. O. Costa, P. H. R. Barbosa, F. F. Barbosa Filho, M. S. Vasconcelos, E. L. Albuquerque, Solid States Commun. 150, 2325 (2011).

40 J. Kaczer and L. Murtinova, phys. stat. sol. (a) 23, 79 (1974).

41 M. Sokolovsky and M. Krawczyk, Journal of Nanoparticle Research 13, 6085 (2011). 
42 T. Wolfram and R. E. De Wames, Phys. Rev. B 8, 3125 (1971).

43 J. W. Kłos, M. Sokolovskyy, S. Mamica, M. Krawczyk, J. Appl. Phys. 111, 123910 (2012).

44 C. A. F. Vaz, J. A. C. Bland, and G. Lauhoff, Rep. Prog. Phys. 71, 056501 (2008). 


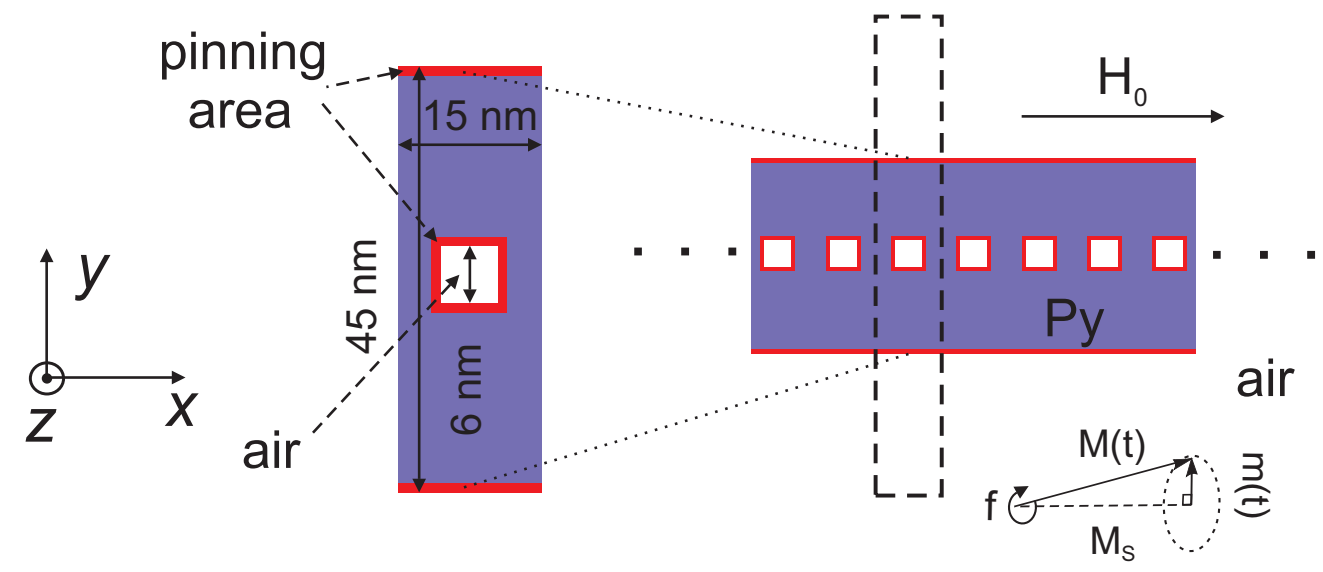

FIG. 1: (color online) Magnonic antidot waveguide under investigation: a $3 \mathrm{~nm}$ thick and $45 \mathrm{~nm}$ wide infinite Py stripe with a periodic series of $6 \mathrm{~nm} \times 6 \mathrm{~nm}$ square antidots disposed centrally along the $x$-axis with a period of a $=15 \mathrm{~nm}$. Bias magnetic field $\mu_{0} H_{0}=1.01 \mathrm{~T}$ is oriented along the $x$-axis. The $1.5 \mathrm{~nm}$ wide red lines at the Py/air interfaces mark the regions in which pinning is assumed in the OOMMF calculations. The dashed box shows the supercell size used in PWM calculations. 

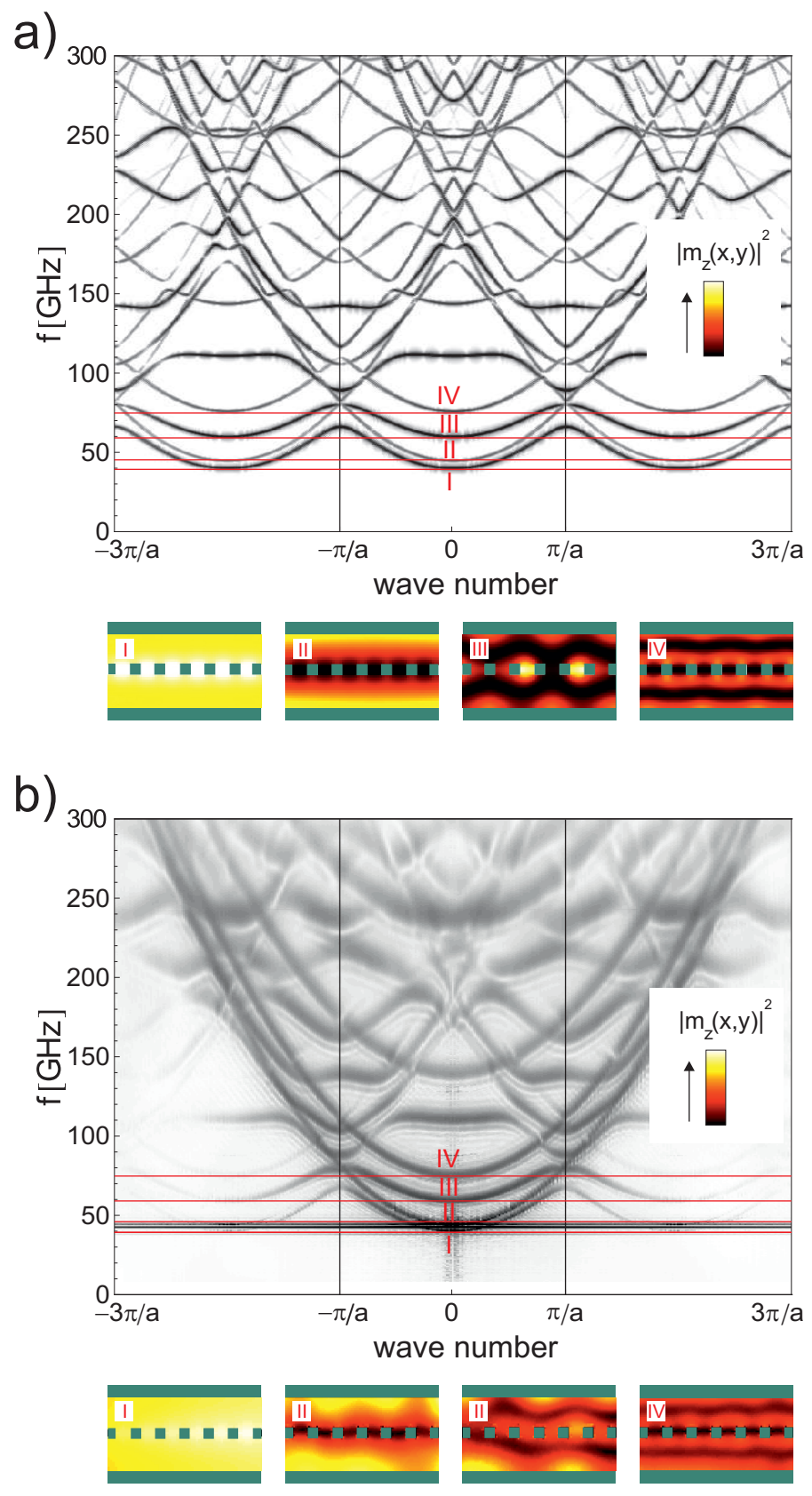

FIG. 2: (color online) Dispersion of spin waves in the MAW presented in Fig. 1, as calculated with (a) OOMMF and (b) Nmag. The vertical lines delimit the $1^{\text {st }}$ Brillouin zone. The magnetization is assumed to precess freely at the Py/air interface, i.e., unpinned magnetization. Bottom in (a) and (b): maps of $\left|m_{\mathrm{z}}(x, y)\right|^{2}$ for the different values of frequency (I to IV) calculated with (a) OOMMF (b) Nmag. Note that the each distribution of $\left|m_{\mathrm{z}}(x, y)\right|^{2}$ obtained by micromagnetic calculations contains contributions of the eigenmodes differing in the wave numbers. 

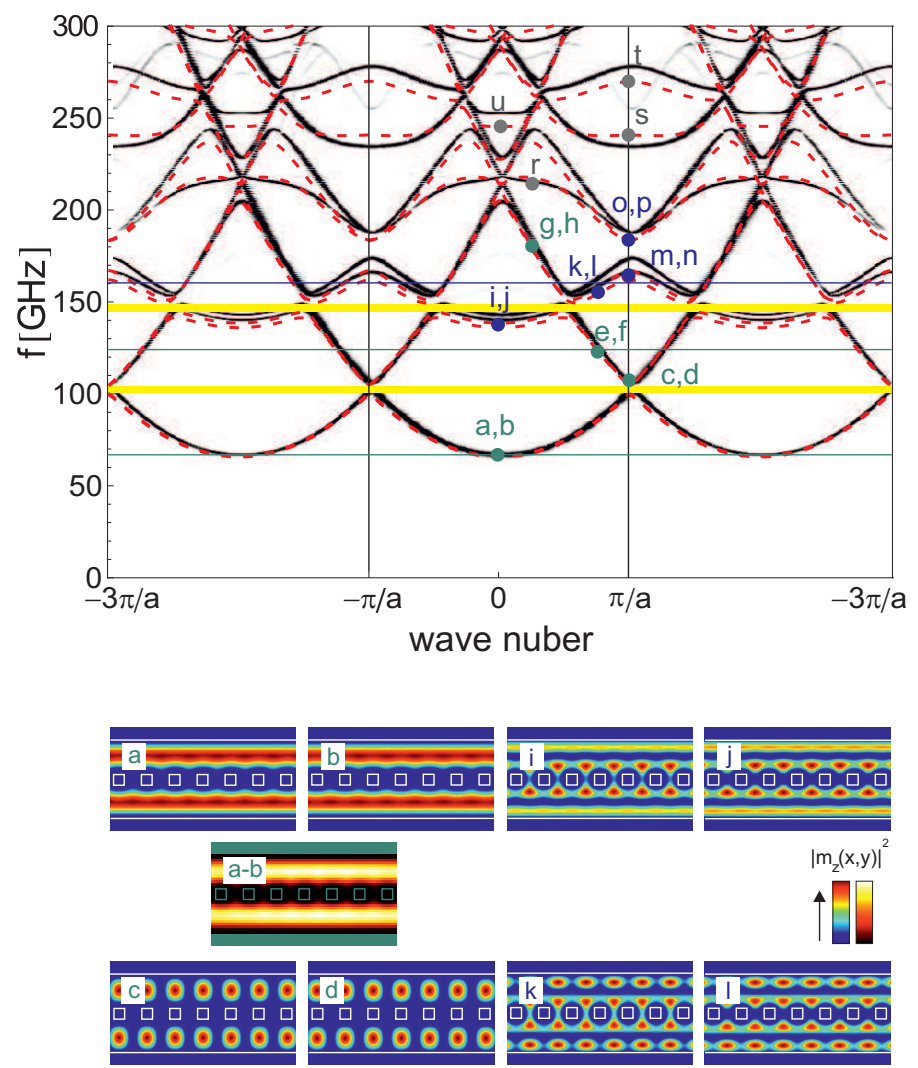

e-f 900000

0000009
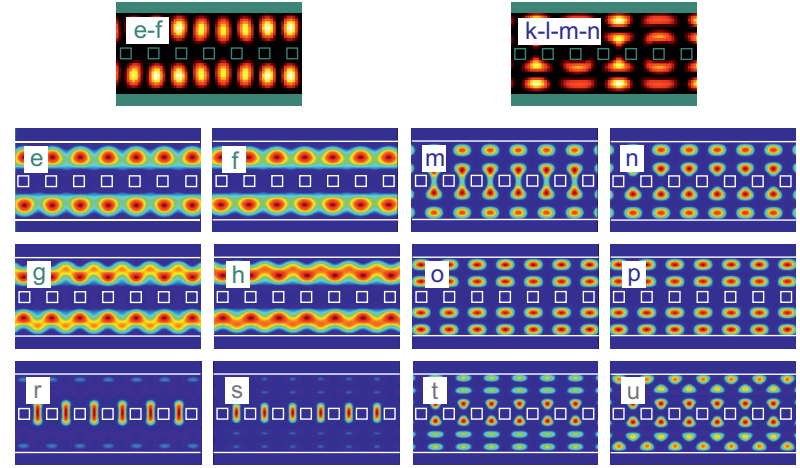

FIG. 3: (color online) Dispersion of spin waves in the MAW presented in Fig. 1, as calculated with OOMMF with dynamic magnetization pinned at the Py/air interfaces (gray lines). PWM results are plotted with red dashed lines. Yellow bars represent the magnonic gaps (in OOMMF calculations). Bottom: maps of $\left|m_{\mathrm{z}}(x, y)\right|^{2}$ at points (a) to (u) in the plot above. Green (a-h) and blue (i-p) labels refer to modes originating from the first and second dispersion parabolas, respectively, of each isolated sub-waveguide at the right and left of the central row of antidots. Brown labels denote high-frequency modes localized in the row of antidots. The maps plotted in full colors scale and hot colors scale present the results calculated with OOMMF and Nmag respectively. The horizontal color lines in dispersion plot mark the contributions from different Bloch bands to the OOMMF profiles. 